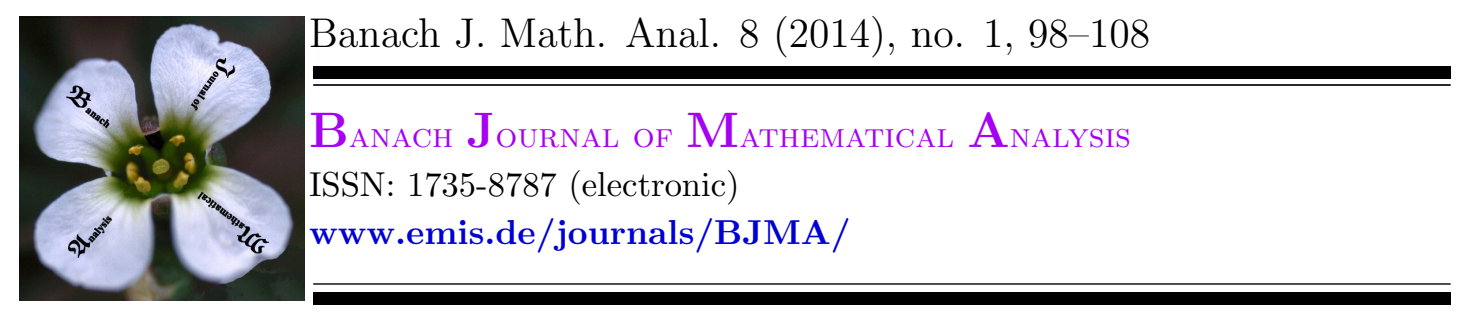

\title{
$(P, Q)$-OUTER GENERALIZED INVERSE OF BLOCK MATRICES IN BANACH ALGEBRAS
}

\author{
MILICA Z. KOLUNDŽIJA \\ Communicated by A. R. Villena
}

ABstraCt. We investigate additive results for $(p, q)$-outer generalized inverse of elements in Banach algebra, along with the representation of this inverse in a block matrix in the Banachiewicz-Schur form. Additionally, we investigate the $(p, q)$-pseudospectrum and $(p, q)$-condition spectrum of a block matrix $x=$ $\left[\begin{array}{ll}a & 0 \\ 0 & b\end{array}\right]_{u}$ in a Banach algebra.

\section{INTRODUCTION}

Let $\mathcal{A}$ be the complex unital Banach algebra with unit 1 . The sets of all idempotents and invertible elements of $\mathcal{A}$ will be denoted by $\mathcal{A}^{\bullet}$ and $\mathcal{A}^{-1}$, respectively.

An element $a \in \mathcal{A}$ is outer generalized invertible, if there exists some $b \in \mathcal{A}$ satisfying $b=b a b$. Such $b$ is called the outer generalized inverse of $a$. In this case $b a$ and $1-a b$ are idempotents corresponding to $a$ and $b$. The set of all outer generalized invertible elements of $\mathcal{A}$ will be denoted with $\mathcal{A}^{(2)}$.

Djordjević and Wei introduced outer generalized inverses with prescribed idempotents in [3] (see also [2]):

Definition 1.1. [3] Let $a \in \mathcal{A}$ and $p, q \in \mathcal{A}^{\bullet}$. An element $b \in \mathcal{A}$ satisfying

$$
b a b=b, b a=p, 1-a b=q,
$$

will be called a $(p, q)$-outer generalized inverse of $a$, written $a_{p, q}^{(2)}=b$.

Date: Received: 1 April 2013; Accepted: 28 April 2013.

2010 Mathematics Subject Classification. Primary 15A09; Secondary 47A05, 46H05, 47A10.

Key words and phrases. $(p, q)$-outer generalized inverse, block matrix, pseudospectrum, condition spectrum. 
The uniqueness of $a_{p, q}^{(2)}$ is provided in the following theorem.

Theorem 1.2. [3] Let $a \in \mathcal{A}$ and $p, q \in \mathcal{A}^{\bullet}$. Then the following statements are equivalent:

(1) $a_{p, q}^{(2)}$ exists;

(2) $(1-q) a=(1-q) a p$, and there exists some $b \in \mathcal{A}$ such that $p b=b, b q=0$ and $a b=1-q$.

Moreover, if $a_{p, q}^{(2)}$ exists, then it is unique.

The set of all outer generalized invertible elements of $\mathcal{A}$ with prescribed idempotents $p, q \in \mathcal{A}^{\bullet}$ will be denoted with $\mathcal{A}_{p, q}^{(2)}$. Outer generalized inverses in rings can be found in [6]. Also, inner generalized inverses with prescribed idempotents were studied in [5].

Let $M$ be a $2 \times 2$ block matrix $M=\left[\begin{array}{ll}A & B \\ C & D\end{array}\right]$, where $A \in \mathbb{C}^{m \times n}, B \in \mathbb{C}^{m \times k}$, $C \in \mathbb{C}^{l \times n}$ and $D \in \mathbb{C}^{l \times k}$. If $A$ is invertible, then the Schur complement of $A$ in $M$ is defined as

$$
S=D-C A^{-1} B
$$

If $\mathrm{M}$ is invertible, then $S$ is invertible, too, and $M$ can be decomposed as

$$
M=\left[\begin{array}{cc}
I_{m} & 0 \\
C A^{-1} & I_{l}
\end{array}\right]\left[\begin{array}{cc}
A & 0 \\
0 & S
\end{array}\right]\left[\begin{array}{cc}
I_{m} & A^{-1} B \\
0 & I_{l}
\end{array}\right]
$$

where $I_{t}$ is the identity matrix of order $t$. In this case, the inverse of $M$ can be written as

$$
\begin{aligned}
M^{-1} & =\left[\begin{array}{cc}
I_{m} & -A^{-1} B \\
0 & I_{l}
\end{array}\right]\left[\begin{array}{cc}
A^{-1} & 0 \\
0 & S^{-1}
\end{array}\right]\left[\begin{array}{cc}
I_{m} & 0 \\
-C A^{-1} & I_{l}
\end{array}\right] \\
& =\left[\begin{array}{cc}
A^{-1}+A^{-1} B S^{-1} C A^{-1} & -A^{-1} B S^{-1} \\
-S^{-1} C A^{-1} & S^{-1}
\end{array}\right] .
\end{aligned}
$$

Result (1.1) is well known as the Banachiewicz-Schur form of $M$, and it has been used in dealing with inverses of block matrices; see [1, 8, 10].

Analogously, we can represent an element of Banach algebra in a block matrix form as follows.

Let $u \in \mathcal{A}$ be an idempotent. Then we can represent element $a \in \mathcal{A}$ as

$$
a=\left[\begin{array}{ll}
a_{11} & a_{12} \\
a_{21} & a_{22}
\end{array}\right]_{u}
$$

where $a_{11}=u a u, a_{12}=u a(1-u), a_{21}=(1-u) a u, a_{22}=(1-u) a(1-u)$.

Let $x=\left[\begin{array}{ll}a & b \\ c & d\end{array}\right]_{u} \in \mathcal{A}$ relative to the idempotent $u \in \mathcal{A}$. If $a \in(u \mathcal{A} u)^{-1}$ and the Schur complement $s=d-c a^{-1} b \in((1-u) \mathcal{A}(1-u))^{-1}$, then the inverse of $x$ has the Banachiewicz-Schur form

$$
x^{-1}=\left[\begin{array}{cc}
a^{-1}+a^{-1} b s^{-1} c a^{-1} & -a^{-1} b s^{-1} \\
-s^{-1} c a^{-1} & s^{-1}
\end{array}\right] \text {. }
$$


If $a \in(u \mathcal{A} u)$ is not invertible but has the outer generalized inverse with prescribed idempotents $p_{1}, q_{1} \in(u \mathcal{A} u)^{\bullet}$, we can observe the generalized Schur complement $s=d-c a_{p_{1}, q_{1}}^{(2)} b$.

Accordingly, we investigate equivalent conditions under which $x_{p, q}^{(2)}$ has the generalized Banachiewicz-Schur form in a Banach algebra.

We use the following auxiliary results.

Lemma 1.3. Let $p, q$ be idempotents in a Banach algebra $\mathcal{A}$. The following statements are equivalent:

(i) $p+q \in \mathcal{A}^{\bullet}$,

(ii) $p q=q p=0$.

Proof. $(i) \Rightarrow(i i)$ : Suppose $p+q \in \mathcal{A}^{\bullet}$. We have

$$
(p+q)^{2}=p+q \Rightarrow p q+q p=0 \Rightarrow p q=-q p .
$$

Since the following holds

$$
p q=p^{2} q^{2}=p(p q) q=p(-q p) q=-p q(p q)=p q q p=p q p=-p p q=-p q,
$$

we obtain $p q=0$. The analogous proof holds for $q p=0$.

$(i i) \Rightarrow(i)$ : Let $p, q \in \mathcal{A}^{\bullet}$ such that $p q=q p=0$. Then

$$
(p+q)^{2}=p^{2}+p q+q p+q^{2}=p+q,
$$

so $p+q \in \mathcal{A}^{\bullet}$.

If $u \in \mathcal{A}^{\bullet}$, then the product of arbitrary elements from algebra $u \mathcal{A} u$ and $(1-$ $u) \mathcal{A}(1-u)$ is equal to 0 , i.e. for all $a \in u \mathcal{A} u$ and for all $b \in(1-u) \mathcal{A}(1-u)$, we have $a b=0$.

Now, as a corollary of Lemma 1.3, we state the following result.

Lemma 1.4. Let $u \in \mathcal{A}^{\bullet}$. If $p_{1} \in(u \mathcal{A} u)^{\bullet}$ and $p_{2} \in((1-u) \mathcal{A}(1-u))^{\bullet}$, then $p=p_{1}+p_{2} \in \mathcal{A}$ is an idempotent.

\section{2. $(p, q)$-OUTER GENERALIZED INVERSE}

The first result gives the additive properties of the $(p, q)$-outer generalized inverse.

Theorem 2.1. Let $p, q \in \mathcal{A}^{\bullet}$ and $a, b \in \mathcal{A}_{p, q}^{(2)}$. If

$$
a_{p, q}^{(2)} b+b_{p, q}^{(2)} a+1=0, \quad a b_{p, q}^{(2)}+b a_{p, q}^{(2)}+1=0,
$$

then $a+b \in \mathcal{A}_{p, q}^{(2)}$ and

$$
(a+b)_{p, q}^{(2)}=a_{p, q}^{(2)}+b_{p, q}^{(2)}
$$


Proof. Using the fact that $a, b \in \mathcal{A}_{p, q}^{(2)}$, Theorem 1.2 and conditions (2.1), we have

$$
\begin{aligned}
& \left(a_{p, q}^{(2)}+b_{p, q}^{(2)}\right)(a+b)\left(a_{p, q}^{(2)}+b_{p, q}^{(2)}\right)= \\
& =a_{p, q}^{(2)}+p b_{p, q}^{(2)}+a_{p, q}^{(2)} b a_{p, q}^{(2)}+a_{p, q}^{(2)}(1-q)+b_{p, q}^{(2)}(1-q)+b_{p, q}^{(2)} a b_{p, q}^{(2)}+p a_{p, q}^{(2)}+b_{p, q}^{(2)} \\
& =a_{p, q}^{(2)}+b_{p, q}^{(2)}+a_{p, q}^{(2)} b a_{p, q}^{(2)}+a_{p, q}^{(2)}+b_{p, q}^{(2)}+b_{p, q}^{(2)} a b_{p, q}^{(2)}+a_{p, q}^{(2)}+b_{p, q}^{(2)} \\
& =a_{p, q}^{(2)}+b_{p, q}^{(2)}+a_{p, q}^{(2)}\left(b a_{p, q}^{(2)}+1\right)+b_{p, q}^{(2)}\left(1+a b_{p, q}^{(2)}\right)+a_{p, q}^{(2)}+b_{p, q}^{(2)} \\
& =a_{p, q}^{(2)}+b_{p, q}^{(2)}+a_{p, q}^{(2)}\left(-a b_{p, q}^{(2)}\right)+b_{p, q}^{(2)}\left(-b a_{p, q}^{(2)}\right)+a_{p, q}^{(2)}+b_{p, q}^{(2)} \\
& =a_{p, q}^{(2)}+b_{p, q}^{(2)}-p b_{p, q}^{(2)}-p a_{p, q}^{(2)}+a_{p, q}^{(2)}+b_{p, q}^{(2)} \\
& =a_{p, q}^{(2)}+b_{p, q}^{(2)} \text {, } \\
& \begin{aligned}
\left(a_{p, q}^{(2)}+b_{p, q}^{(2)}\right)(a+b) & =a_{p, q}^{(2)} a+a_{p, q}^{(2)} b+b_{p, q}^{(2)} a+b_{p, q}^{(2)} b \\
& =p+p a_{p, q}^{(2)} b+p b_{p, q}^{(2)} a+p \\
& =p+p\left(a_{p, q}^{(2)} b+b_{p, q}^{(2)} a+1\right) \\
& =p
\end{aligned}
\end{aligned}
$$

and also

$$
\begin{aligned}
(a+b)\left(a_{p, q}^{(2)}+b_{p, q}^{(2)}\right) & =a a_{p, q}^{(2)}+b a_{p, q}^{(2)}+a b_{p, q}^{(2)}+b b_{p, q}^{(2)} \\
& =(1-q)+b a_{p, q}^{(2)}+a b_{p, q}^{(2)}+(1-q) \\
& =(1-q)+b a_{p, q}^{(2)}(1-q)+a b_{p, q}^{(2)}(1-q)+(1-q) \\
& =(1-q)+\left(b a_{p, q}^{(2)}+a b_{p, q}^{(2)}+1\right)(1-q) \\
& =(1-q) .
\end{aligned}
$$

Thus, we proved $(a+b)_{p, q}^{(2)}=a_{p, q}^{(2)}+b_{p, q}^{(2)}$.

The following theorem gives us equivalent conditions under which $x_{p, q}^{(2)}$ has the generalized Banachiewicz-Schur form in a Banach algebra.

Theorem 2.2. Let $x=\left[\begin{array}{ll}a & b \\ c & d\end{array}\right]_{u} \in \mathcal{A}$ relative to the idempotent $u \in \mathcal{A}, p_{1}, q_{1} \in$ $(u \mathcal{A} u)^{\bullet}$ and $p_{2}, q_{2} \in((1-u) \mathcal{A}(1-u))^{\bullet}$ and let $p=p_{1}+p_{2} \in \mathcal{A}$ and $q=q_{1}+q_{2} \in \mathcal{A}$. Let $a \in(u \mathcal{A} u)_{p_{1}, q_{1}}^{(2)}$ and let $s=d-c a_{p_{1}, q_{1}}^{(2)} b \in((1-u) \mathcal{A}(1-u))_{p_{2}, q_{2}}^{(2)}$ be the generalized Schur complement of $a$ in $x$. Then the following statements are equivalent:

(i) $x \in \mathcal{A}_{p, q}^{(2)}$ and $x_{p, q}^{(2)}=r$, where

$$
r=\left[\begin{array}{cc}
a_{p_{1}, q_{1}}^{(2)}+a_{p_{1}, q_{1}}^{(2)} b s_{p_{2}, q_{2}}^{(2)} c a_{p_{1}, q_{1}}^{(2)} & -a_{p_{1}, q_{1}}^{(2)} b s_{p_{2}, q_{2}}^{(2)} \\
-s_{p_{2}, q_{2}}^{(2)} c a_{p_{1}, q_{1}}^{(2)} & s_{p_{2}, q_{2}}^{(2)}
\end{array}\right]
$$

(ii) $c a_{p_{1}, q_{1}}^{(2)} a=s s_{p_{2}, q_{2}}^{(2)} c$ and $a a_{p_{1}, q_{1}}^{(2)} b=b s_{p_{2}, q_{2}}^{(2)} s$.

Proof. By Lemma 1.4 we obtain that $p$ and $q$ are idempotents.

Using the assumptions $a \in(u \mathcal{A} u)_{p_{1}, q_{1}}^{(2)}$ and $s \in((1-u) \mathcal{A}(1-u))_{p_{2}, q_{2}}^{(2)}$, we verify $r x r=r$.

The equation $r x=p$ is equivalent to the equations:

$$
s_{p_{2}, q_{2}}^{(2)} c=s_{p_{2}, q_{2}}^{(2)} c a_{p_{1}, q_{1}}^{(2)} a \quad \text { and } \quad a_{p_{1}, q_{1}}^{(2)} b=a_{p_{1}, q_{1}}^{(2)} b s_{p_{2}, q_{2}}^{(2)} s .
$$


On the other hand, $1-x r=q$ is equivalent to:

$$
b s_{p_{2}, q_{2}}^{(2)}=a a_{p_{1}, q_{1}}^{(2)} b s_{p_{2}, q_{2}}^{(2)} \quad \text { and } \quad c a_{p_{1}, q_{1}}^{(2)}=s s_{p_{2}, q_{2}}^{(2)} c a_{p_{1}, q_{1}}^{(2)} .
$$

Therefore, $x$ has $(p, q)$-outer generalized inverse if and only if

$$
\begin{aligned}
& s_{p_{2}, q_{2}}^{(2)} c=s_{p_{2}, q_{2}}^{(2)} c a_{p_{1}, q_{1}}^{(2)} a, \quad a_{p_{1}, q_{1}}^{(2)} b=a_{p_{1}, q_{1}}^{(2)} b s_{p_{2}, q_{2}}^{(2)} s, \\
& b s_{p_{2}, q_{2}}^{(2)}=a a_{p_{1}, q_{1}}^{(2)} b s_{p_{2}, q_{2}}^{(2)}, \quad c a_{p_{1}, q_{1}}^{(2)}=s s_{p_{2}, q_{2}}^{(2)} c a_{p_{1}, q_{1}}^{(2)},
\end{aligned}
$$

which are equivalent to

$$
c a_{p_{1}, q_{1}}^{(2)} a=s s_{p_{2}, q_{2}}^{(2)} c, \quad b s_{p_{2}, q_{2}}^{(2)} s=a a_{p_{1}, q_{1}}^{(2)} b .
$$

As a corollary, we formulate the following result.

Corollary 2.3. Let $x=\left[\begin{array}{ll}a & b \\ c & d\end{array}\right] \in \mathcal{A}$ relative to the idempotent $u \in \mathcal{A}, p_{1}, q_{1} \in$ $(u \mathcal{A} u)^{\bullet}$ and $p_{2}, q_{2} \in((1-u) \mathcal{A}(1-u))^{\bullet}$ and let $p=p_{1}+p_{2} \in \mathcal{A}$ and $q=q_{1}+q_{2} \in \mathcal{A}$. Let $a \in(u \mathcal{A} u)_{p_{1}, q_{1}}^{(2)}$ and let $s=d-c a_{p_{1}, q_{1}}^{(2)} b \in((1-u) \mathcal{A}(1-u))_{p_{2}, q_{2}}^{(2)}$. The following statements are equivalent:

(i) $c a_{p_{1}, q_{1}}^{(2)}=a_{p_{1}, q_{1}}^{(2)} b=b s_{p_{2}, q_{2}}^{(2)}=s_{p_{2}, q_{2}}^{(2)} c=0$

(ii) $c a_{p_{1}, q_{1}}^{(2)} a=s s_{p_{2}, q_{2}}^{(2)} c, \quad a a_{p_{1}, q_{1}}^{(2)} b=b s_{p_{2}, q_{2}}^{(2)} s$, $a_{p_{1}, q_{1}}^{(2)} b s_{p_{2}, q_{2}}^{(2)}=s_{p_{2}, q_{2}}^{(2)} c a_{p_{1}, q_{1}}^{(2)}=0$.

If one of these conditions is satisfied, then $x \in \mathcal{A}_{p, q}^{(2)}$ and

$$
x_{p, q}^{(2)}=\left[\begin{array}{cc}
a_{p_{1}, q_{1}}^{(2)}+a_{p_{1}, q_{1}}^{(2)} b s_{p_{2}, q_{2}}^{(2)} c a_{p_{1}, q_{1}}^{(2)} & -a_{p_{1}, q_{1}}^{(2)} b s_{p_{2}, q_{2}}^{(2)} \\
-s_{p_{2}, q_{2}}^{(2)} c a_{p_{1}, q_{1}}^{(2)} & s_{p_{2}, q_{2}}^{(2)}
\end{array}\right] .
$$

\section{3. $(p, q)$-CONDITION SPECTRUM AND $(p, q)$-PSEUdospeCtrum} [9].

The pseudospectrum and the condition spectrum were studied in [4], [7] and

Definition 3.1. [9] (Pseudospectrum)

Let $\epsilon>0$. The $\epsilon$-pseudospectrum of an element $a \in \mathcal{A}$ is defined as

$$
\Lambda_{\epsilon}(a)=\left\{z \in \mathbb{C} \mid a-z \text { is not invertible or }\left\|(a-z)^{-1}\right\| \geq \epsilon\right\} .
$$

Definition 3.2. [4] (Condition spectrum)

Let $0<\epsilon<1$. The $\epsilon$-condition spectrum of an element $a \in \mathcal{A}$ is defined as

$$
\sigma_{\epsilon}(a)=\left\{z \in \mathbb{C} \mid a-z \text { is not invertible or }\left\|(a-z)^{-1}\right\| \cdot\|a-z\| \geq \frac{1}{\epsilon}\right\} .
$$

We generalize the pseudospectrum and the condition spectrum, and we formulate $(p, q)$-pseudospectrum and $(p, q)$-condition spectrum as follows: 
Definition 3.3. $((p, q)$-pseudospectrum)

Let $\epsilon>0$. The $(p, q)-\epsilon$-pseudospectrum of an element $a \in \mathcal{A}$ is defined as

$$
\Lambda_{\epsilon}(a)=\left\{z \in \mathbb{C} \mid a-z \notin \mathcal{A}_{p, q}^{(2)} \text { or }\left\|(a-z)_{p, q}^{(2)}\right\| \geq \epsilon\right\} .
$$

Definition 3.4. $((p, q)$-condition spectrum)

Let $0<\epsilon<1$. The $(p, q)-\epsilon$-condition spectrum of an element $a \in \mathcal{A}$ is defined as

$$
\sigma_{(p, q)-\epsilon}(a)=\left\{z \in \mathbb{C} \mid a-z \notin \mathcal{A}_{p, q}^{(2)} \text { or }\left\|(a-z)_{p, q}^{(2)}|| \cdot\right\| a-z \| \geq \frac{1}{\epsilon}\right\} .
$$

Notice that the uniqueness of $a_{p, q}^{(2)}$ allows us to consider the $(p, q)$-pseudospectrum and $(p, q)$-condition spectrum.

If $x=\left[\begin{array}{ll}a & 0 \\ 0 & b\end{array}\right]_{u} \in \mathcal{A}$ relative to the idempotent $u \in \mathcal{A}$, then the norm of $x$ can be define as

$$
\|x\|=\max \{\|a\|,\|b\|\} .
$$

Now, we state an auxiliary result.

Lemma 3.5. Let $x=\left[\begin{array}{ll}a & 0 \\ 0 & b\end{array}\right]_{u} \in \mathcal{A}$ relative to the idempotent $u \in \mathcal{A}, p_{1}, q_{1} \in$ $(u \mathcal{A} u)^{\bullet}$ and $p_{2}, q_{2} \in((1-u) \mathcal{A}(1-u))^{\bullet}$ and let $p=p_{1}+p_{2} \in \mathcal{A}$ and $q=q_{1}+q_{2} \in \mathcal{A}$. Then $x \in \mathcal{A}_{p, q}^{(2)}$ if and only if $a \in(u \mathcal{A} u)_{p_{1}, q_{1}}^{(2)}$ and $b \in((1-u) \mathcal{A}(1-u))_{p_{2}, q_{2}}^{(2)}$. If $x \in \mathcal{A}_{p, q}^{(2)}$, then

$$
x_{p, q}^{(2)}=\left[\begin{array}{cc}
a_{p_{1}, q_{1}}^{(2)} & 0 \\
0 & b_{p_{2}, q_{2}}^{(2)}
\end{array}\right]_{u} .
$$

Proof. By Lemma 1.4 we obtain that $p$ and $q$ are idempotents.

If $a \in(u \mathcal{A} u)_{p_{1}, q_{1}}^{(2)}$ and $b \in((1-u) \mathcal{A}(1-u))_{p_{2}, q_{2}}^{(2)}$, by Theorem 2.2, we obtain $x \in \mathcal{A}_{p, q}^{(2)}$.

If $x \in \mathcal{A}_{p, q}^{(2)}$, there exists the element $y=\left[\begin{array}{cc}a_{1} & c \\ d & b_{1}\end{array}\right]_{u} \in \mathcal{A}$ such that $y=x_{p, q}^{(2)}$. The equation $y x y=y$ is equivalent to equations:

$$
\begin{array}{r}
a_{1} a a_{1}+c b d=a_{1} \\
a_{1} a c+c b b_{1}=c \\
d a a_{1}+b_{1} b d=d \\
d a c+b_{1} b b_{1}=b_{1} .
\end{array}
$$

Also, $y x=p$ is equivalent to:

$$
\begin{gathered}
a_{1} a=p_{1} \\
c b=0 \\
d a=0 \\
b_{1} b=p_{2},
\end{gathered}
$$


and $1-x y=q$ is equivalent to:

$$
\begin{array}{r}
u-a a_{1}=q_{1} \\
a c=0 \\
b d=0 \\
(1-u)-b b_{1}=q_{2} .
\end{array}
$$

The equations $a_{1} a c+c b b_{1}=c, c b=0$ and $a c=0$ imply $c=0$. Analogously, $d a a_{1}+b_{1} b d=d, d a=0$ and $b d=0$ imply $d=0$. Now, we have the equations:

$$
\begin{array}{r}
a_{1} a a_{1}=a_{1} \\
a_{1} a=p_{1} \\
u-a a_{1}=q_{1},
\end{array}
$$

and

$$
\begin{aligned}
b_{1} b b_{1} & =b_{1} \\
b_{1} b & =p_{2} \\
(1-u)-b b_{1} & =q_{2}
\end{aligned}
$$

proving $a_{1}=a_{p_{1}, q_{1}}^{(2)}$ and $b_{1}=b_{p_{2}, q_{2}}^{(2)}$.

Furthermore, if $x \in \mathcal{A}_{p, q}^{(2)}$, then

$$
x_{p, q}^{(2)}=\left[\begin{array}{cc}
a_{p_{1}, q_{1}}^{(2)} & 0 \\
0 & b_{p_{2}, q_{2}}^{(2)}
\end{array}\right]_{u} .
$$

As a corollary, we have the following result for the invertibility of an element $x=\left[\begin{array}{ll}a & 0 \\ 0 & b\end{array}\right]_{u} \in \mathcal{A}$ relative to the idempotent $u \in \mathcal{A}$.

Lemma 3.6. Let $x=\left[\begin{array}{ll}a & 0 \\ 0 & b\end{array}\right]_{u} \in \mathcal{A}$ relative to the idempotent $u \in \mathcal{A}$. Then $x \in \mathcal{A}^{-1}$ if and only if $a \in(u \mathcal{A} u)^{-1}$ and $b \in((1-u) \mathcal{A}(1-u))^{-1}$.

If $x \in \mathcal{A}^{-1}$, then

$$
x^{-1}=\left[\begin{array}{cc}
a^{-1} & 0 \\
0 & b^{-1}
\end{array}\right]_{u}
$$

Therefore, for the spectrum of an element $x=\left[\begin{array}{ll}a & 0 \\ 0 & b\end{array}\right]_{u} \in \mathcal{A}$, the following holds

$$
\sigma(x)=\sigma(a) \cup \sigma(b) .
$$

We investigate whether the similar property holds for the pseudospectrum and condition spectrum. We formulate the following results. 
Theorem 3.7. Let $x=\left[\begin{array}{ll}a & 0 \\ 0 & b\end{array}\right]_{u} \in \mathcal{A}$ relative to the idempotent $u \in \mathcal{A}, \epsilon>0$, $p_{1}, q_{1} \in(u \mathcal{A} u)^{\bullet}$ and $p_{2}, q_{2} \in((1-u) \mathcal{A}(1-u))^{\bullet}$ and let $p=p_{1}+p_{2} \in \mathcal{A}$ and $q=q_{1}+q_{2} \in \mathcal{A}$. Then

$$
\Lambda_{(p, q)-\epsilon}(x)=\Lambda_{\left(p_{1}, q_{1}\right)-\epsilon}(a) \cup \Lambda_{\left(p_{2}, q_{2}\right)-\epsilon}(b) .
$$

Proof. Let $z \in \Lambda_{(p, q)-\epsilon}(x)$. Then $x-z \notin \mathcal{A}_{p, q}^{(2)}$ or $\left\|(x-z)_{p, q}^{(2)}\right\| \geq \epsilon$.

If $x-z=\left[\begin{array}{cc}a-z u & 0 \\ 0 & b-z(1-u)\end{array}\right]_{u} \notin \mathcal{A}_{p, q}^{(2)}$, by Lemma 3.5, we obtain that $a-z u \notin(u \mathcal{A} u)_{p_{1}, q_{1}}^{(2)}$ or $b-z(1-u) \notin((1-u) \mathcal{A}(1-u))_{p_{2}, q_{2}}^{(2)}$. It implies $z \in$ $\Lambda_{\left(p_{1}, q_{1}\right)-\epsilon}(a)$ or $z \in \Lambda_{\left(p_{2}, q_{2}\right)-\epsilon}(b)$, so $z \in \Lambda_{\left(p_{1}, q_{1}\right)-\epsilon}(a) \cup \Lambda_{\left(p_{2}, q_{2}\right)-\epsilon}(b)$.

If $x-z=\left[\begin{array}{cc}a-z u & 0 \\ 0 & b-z(1-u)\end{array}\right]_{u} \in \mathcal{A}_{p, q}^{(2)}$, we have

$$
(x-z)_{p, q}^{(2)}=\left[\begin{array}{cc}
(a-z u)_{p_{1}, q_{1}}^{(2)} & 0 \\
0 & (b-z(1-u))_{p_{2}, q_{2}}^{(2)}
\end{array}\right]_{u}
$$

and

$$
\left\|(x-z)_{p, q}^{(2)}\right\|=\max \left\{\left\|(a-z u)_{p_{1}, q_{1}}^{(2)}\right\|,\left\|(b-z(1-u))_{p_{2}, q_{2}}^{(2)}\right\|\right\} \geq \epsilon .
$$

By Lemma 3.5, we conclude that

$$
a-z u \in(u \mathcal{A} u)_{p_{1}, q_{1}}^{(2)} \text { and } b-z(1-u) \in((1-u) \mathcal{A}(1-u))_{p_{2}, q_{2}}^{(2)} .
$$

The assumption $\max \left\{\left\|(a-z u)_{p_{1}, q_{1}}^{(2)}\right\|,\left\|(b-z(1-u))_{p_{2}, q_{2}}^{(2)}\right\|\right\} \geq \epsilon$ implies that either $\left\|(a-z u)_{p_{1}, q_{1}}^{(2)}\right\| \geq \epsilon$ or $\left\|(b-z(1-u))_{p_{2}, q_{2}}^{(2)}\right\| \geq \epsilon$ holds. It follows that $z \in \Lambda_{\left(p_{1}, q_{1}\right)-\epsilon}(a)$ or $z \in \Lambda_{\left(p_{2}, q_{2}\right)-\epsilon}(b)$, so $z \in \Lambda_{\left(p_{1}, q_{1}\right)-\epsilon}(a) \cup \Lambda_{\left(p_{2}, q_{2}\right)-\epsilon}(b)$.

We have proved $\Lambda_{(p, q)-\epsilon}(x) \subset \Lambda_{\left(p_{1}, q_{1}\right)-\epsilon}(a) \cup \Lambda_{\left(p_{2}, q_{2}\right)-\epsilon}(b)$.

Now, let $z \in \Lambda_{\left(p_{1}, q_{1}\right)-\epsilon}(a) \cup \Lambda_{\left(p_{2}, q_{2}\right)-\epsilon}(b)$. It follows

$$
a-z u \notin(u \mathcal{A} u)_{p_{1}, q_{1}}^{(2)} \text { or }\left\|(a-z u)_{p_{1}, q_{1}}^{(2)}\right\| \geq \epsilon
$$

or

$$
b-z(1-u) \notin((1-u) \mathcal{A}(1-u))_{p_{2}, q_{2}}^{(2)} \text { or }\left\|(b-z(1-u))_{p_{2}, q_{2}}^{(2)}\right\| \geq \epsilon .
$$

If either $a-z u \notin(u \mathcal{A} u)_{p_{1}, q_{1}}^{(2)}$ or $b-z(1-u) \notin((1-u) \mathcal{A}(1-u))_{p_{2}, q_{2}}^{(2)}$, by Lemma 3.5 , it follows $x-z \notin \mathcal{A}_{p, q}^{(2)}$. So, $z \in \Lambda_{(p, q)-\epsilon}(x)$.

On the other hand, if

$$
a-z u \in(u \mathcal{A} u)_{p_{1}, q_{1}}^{(2)} \text { and } b-z(1-u) \in((1-u) \mathcal{A}(1-u))_{p_{2}, q_{2}}^{(2)},
$$

it holds either $\left\|(a-z u)_{p_{1}, q_{1}}^{(2)}\right\| \geq \epsilon$ or $\left\|(b-z(1-u))_{p_{2}, q_{2}}^{(2)}\right\| \geq \epsilon$. Therefore, $\left\|(x-z)_{p, q}^{(2)}\right\|=\max \left\{\left\|(a-z u)_{p_{1}, q_{1}}^{(2)}\right\|,\left\|(b-z(1-u))_{p_{2}, q_{2}}^{(2)}\right\|\right\} \geq \epsilon$. This proves that $z \in \Lambda_{(p, q)-\epsilon}(x)$.

The inclusion $\Lambda_{\left(p_{1}, q_{1}\right)-\epsilon}(a) \cup \Lambda_{\left(p_{2}, q_{2}\right)-\epsilon}(b) \subset \Lambda_{(p, q)-\epsilon}(x)$ has been proved. 
Theorem 3.8. Let $x=\left[\begin{array}{ll}a & 0 \\ 0 & b\end{array}\right]_{u} \in \mathcal{A}$ relative to the idempotent $u \in \mathcal{A}, 0<\epsilon<$ $1, p_{1}, q_{1} \in(u \mathcal{A} u)^{\bullet}$ and $p_{2}, q_{2} \in((1-u) \mathcal{A}(1-u))^{\bullet}$ and let $p=p_{1}+p_{2} \in \mathcal{A}$ and $q=q_{1}+q_{2} \in \mathcal{A}$. Then

$$
\sigma_{\left(p_{1}, q_{1}\right)-\epsilon}(a) \cup \sigma_{\left(p_{2}, q_{2}\right)-\epsilon}(b) \subset \sigma_{(p, q)-\epsilon}(x) .
$$

Proof. Let $z \in \sigma_{\left(p_{1}, q_{1}\right)-\epsilon}(a) \cup \sigma_{\left(p_{2}, q_{2}\right)-\epsilon}(b)$. These imply

$$
a-z u \notin(u \mathcal{A} u)_{p_{1}, q_{1}}^{(2)} \text { or }\left\|(a-z u)_{p_{1}, q_{1}}^{(2)}\right\| \cdot\|a-z u\| \geq \frac{1}{\epsilon}
$$

or

$b-z(1-u) \notin((1-u) \mathcal{A}(1-u))_{p_{2}, q_{2}}^{(2)}$ or $\left\|(b-z(1-u))_{p_{2}, q_{2}}^{(2)}\right\| \cdot\|b-z(1-u)\| \geq \frac{1}{\epsilon}$.

If either $a-z u \notin(u \mathcal{A} u)_{p_{1}, q_{1}}^{(2)}$ or $b-z(1-u) \notin((1-u) \mathcal{A}(1-u))_{p_{2}, q_{2}}^{(2)}$, by Lemma 3.5 , it follows $x-z \notin \mathcal{A}_{p, q}^{(2)}$. Then, we have $z \in \sigma_{(p, q)-\epsilon}(x)$.

On the other hand, if

$$
a-z u \in(u \mathcal{A} u)_{p_{1}, q_{1}}^{(2)} \text { and } b-z(1-u) \in((1-u) \mathcal{A}(1-u))_{p_{2}, q_{2}}^{(2)},
$$

it holds either

$$
\left\|(a-z u)_{p_{1}, q_{1}}^{(2)}\right\| \cdot\|a-z u\| \geq \frac{1}{\epsilon} \text { or }\left\|(b-z(1-u))_{p_{2}, q_{2}}^{(2)}\right\| \cdot\|b-z(1-u)\| \geq \frac{1}{\epsilon} .
$$

Without loss of generality, assume that $\left\|(a-z u)_{p_{1}, q_{1}}^{(2)}\right\| \cdot\|a-z u\| \geq \frac{1}{\epsilon}$ holds. Therefore,

$$
\begin{aligned}
& \left\|(x-z)_{p, q}^{(2)} \mid\right\|\|x-z\|= \\
& =\max \left\{\left\|(a-z u)_{p_{1}, q_{1}}^{(2)}\right\|,\left\|(b-z(1-u))_{p_{2}, q_{2}}^{(2)}\right\|\right\} \cdot \max \{\|a-z u\|,\|b-z(1-u)\|\} \\
& \geq\left\|(a-z u)_{p_{1}, q_{1}}^{(2)}\right\| \cdot\|a-z u\| \geq \frac{1}{\epsilon} .
\end{aligned}
$$

This proves that $z \in \sigma_{(p, q)-\epsilon}(x)$.

The next example shows that the converse inclusion is not true in the previous theorem.

Example 3.9. Let $0<\epsilon<1, z \in \mathbb{C}$ and $u \in \mathcal{A}^{\bullet}$ such that $\|u\|<\frac{1}{\sqrt{\epsilon}}$ and $\|1-u\|<\frac{1}{\sqrt{\epsilon}}$. Let $x=\left[\begin{array}{cc}\left(\epsilon^{2}+z\right) u & 0 \\ 0 & (\epsilon+z)(1-u)\end{array}\right]_{u} \in \mathcal{A}$ relative to the idempotent $u \in \mathcal{A}$. Then

$$
z \in \sigma_{(1,0)-\epsilon}(x) \text {, but } z \notin\left(\sigma_{(u, 0)-\epsilon}\left(\left(\epsilon^{2}+z\right) u\right) \cup \sigma_{(1-u, 0)-\epsilon}((\epsilon+z)(1-u))\right) .
$$

Proof. For idempotents $u \in \mathcal{A}$ and $1-u \in \mathcal{A}$, we have $\|u\| \geq 1$ and $\|1-u\| \geq 1$. There exists the inverse

$$
(x-z)_{1,0}^{(2)}=\left[\begin{array}{cc}
\frac{1}{\epsilon^{2}} u & 0 \\
0 & \frac{1}{\epsilon}(1-u)
\end{array}\right]_{u}
$$


as well as inverses

$$
\left(\left(\epsilon^{2}+z\right) u-z u\right)_{u, 0}^{(2)}=\left(\epsilon^{2} u\right)_{u, 0}^{(2)}=\frac{1}{\epsilon^{2}} u
$$

and

$$
((\epsilon+z)(1-u)-z(1-u))_{1-u, 0}^{(2)}=(\epsilon(1-u))_{1-u, 0}^{(2)}=\frac{1}{\epsilon}(1-u) .
$$

Now, we have

$$
\begin{aligned}
& \left\|(x-z)_{1,0}^{(2)} \mid\right\|\|x-z\|= \\
& =\max \left\{\left\|\frac{1}{\epsilon^{2}} u\right\|,\left\|\frac{1}{\epsilon}(1-u) \mid\right\|\right\} \cdot \max \left\{\left\|\epsilon^{2} u\right\|,\|\epsilon(1-u)\|\right\} \\
& =\left\|\frac{1}{\epsilon^{2}} u\right\| \cdot\|\epsilon(1-u)\| \geq\left|\frac{1}{\epsilon^{2}}\right| \cdot|\epsilon| \geq \frac{1}{\epsilon}
\end{aligned}
$$

but also

$$
\left\|\left(\epsilon^{2} u\right)_{u, 0}^{(2)}\right\| \cdot\left\|\epsilon^{2} u\right\|=\left\|\frac{1}{\epsilon^{2}} u\right\| \cdot\left\|\epsilon^{2} u\right\|=\|u\|^{2}<\frac{1}{\epsilon}
$$

and

$$
\left\|(\epsilon(1-u))_{1-u, 0}^{(2)}\right\| \cdot\|\epsilon(1-u)\|=\left\|\frac{1}{\epsilon}(1-u)\right\| \cdot\|\epsilon(1-u)\|=\|1-u\|^{2}<\frac{1}{\epsilon} .
$$

Therefore,

$$
z \in \sigma_{(1,0)-\epsilon}(x), \text { but } z \notin\left(\sigma_{(u, 0)-\epsilon}\left(\left(\epsilon^{2}+z\right) u\right) \cup \sigma_{(1-u, 0)-\epsilon}((\epsilon+z)(1-u))\right) .
$$

If $x \in \mathcal{A}$ is invertible, $p=1$ and $q=0$, then $x^{-1}=x_{p, q}^{(2)}$.

As corollaries of Theorem 3.7 and Theorem 3.8, we formulate the following results for the pseudospectrum and the condition spectrum.

Theorem 3.10. Let $x=\left[\begin{array}{ll}a & 0 \\ 0 & b\end{array}\right]_{u} \in \mathcal{A}$ relative to the idempotent $u \in \mathcal{A}$ and $\epsilon>0$. Then

$$
\Lambda_{\epsilon}(x)=\Lambda_{\epsilon}(a) \cup \Lambda_{\epsilon}(b)
$$

Theorem 3.11. Let $x=\left[\begin{array}{ll}a & 0 \\ 0 & b\end{array}\right]_{u} \in \mathcal{A}$ relative to the idempotent $u \in \mathcal{A}$ and $0<\epsilon<1$. Then

$$
\sigma_{\epsilon}(a) \cup \sigma_{\epsilon}(b) \subset \sigma_{\epsilon}(x)
$$

Acknowledgement. The author is supported by the Ministry of Education, Science and Technological Development, Republic of Serbia, grant no. 174007 


\section{REFERENCES}

1. J.K. Baksalary and G.P.H. Styan, Generalized inverses of partitioned matrices in Banachiewicz-Schur form, Linear Algebra Appl. 354 (2002), 41-47.

2. D.S. Djordjevic and V. Rakocevic, Lectures on generalized inverses, Faculty of Sciences and Mathematics, University of Nis, 2008.

3. D.S. Djordjević and Y. Wei, Outer generalized inverses in rings, Comm. Algebra 33 (2005), 3051-3060.

4. S.H. Kulkarni and D. Sukumar, The condition spectrum, Acta Sci. Math. (Szeged) 74 (2008), no. 3-4, 625-641.

5. B. Načevska and D.S. Djordjević, Inner generalized inverses with prescribed idempotents, Comm. Algebra 39 (2011), 1-14.

6. B. Načevska and D.S. Djordjević, Outer generalized inverses in rings and related idempotents, Publ. Math. Debrecen 73 (2008), no. 3-4, 309-316.

7. D. Sukumar, Comparative results on eigenvalues, pseudospectra and conditionspectra, arXiv preprint arXiv: 1109.2731 (2011).

8. Y. Tian and Y. Takane, Schur Complements and Banachiewicz-Schur Forms, Electronic. J. Linear Algebra 13 (2005), 405-418.

9. L.N. Trefethen and M. Embree, Spectra and pseudospectra, Princeton University Press, Princeton, NJ, 2005.

10. F. Zhang (Ed.), The Schur Complement and its Applications, Springer, 2005.

Faculty of Sciences and Mathematics, University of Niš, P.O. Box 224, ViŠEgradska 33, 18000 Niš, SERBIA.

E-mail address: mkolundzija@pmf.ni.ac.rs, milica.kolundzija@gmail.com 